\title{
A Consistent Lattice Boltzmann Equation with Baroclinic Coupling for Mixtures
}

\author{
Pietro Asinari ${ }^{a}$ and Li-Shi Luo ${ }^{b}$ \\ a Department of Energetics, Politecnico di Torino \\ Corso Duca degli Abruzzi 24, Zip Code 10129, Torino, Italy \\ Tel.: +39 (011) 090-4520, FAX: +39 (011) 090-4499 \\ ${ }^{\mathrm{b}}$ Department of Mathematics and Statistics \\ and Center for Computational Sciences \\ Old Dominion University, Norfolk, VA 23529, USA \\ Tel.: +1 (757) 683-5295, FAX: +1 (757) 683-3885
}

\begin{abstract}
We propose a consistent lattice Boltzmann equation (LBE) with baroclinic coupling between species and mixture dynamics to model the active scalar dynamics in multi-species mixtures. The proposed LBE model is directly derived from the linearized Boltzmann equations for mixtures and it has the following two distinctive features. First, it uses the multiple-relaxation-time collision model so that it has the flexibility of independent Reynolds and Schmidt numbers, and better numerical stability. Second, it satisfies the indifferentiability principle therefore leads to a set of consistent hydrodynamic equations for barycentric velocity for mixtures. The proposed LBE model is validated through simulations of decaying homogeneous isotropic turbulence in three dimensions. We simulate both the active and passive scalar dynamics in decaying turbulence for mixtures. We also compute various statistical quantities and their decay exponents in decaying turbulence. Our results agree well with existing results for both scalar dynamics and decaying turbulence.
\end{abstract}

Key words: mixture modeling, turbulence, DNS, lattice Boltzmann method, baroclinic effect

Email addresses: pietro.asinari@polito.it (Pietro Asinari), lluo@odu.edu (Li-Shi Luo). 


\section{Introduction}

Turbulence is a mechanism for effective mixing, and turbulent mixing is relevant to various engineering applications. Turbulent flows generate large interfacial surface areas among interacting species with small eddies produced by turbulent energy cascade, which permits the otherwise slow molecular mixing to proceed faster [1]. Turbulent mixing involves three processes: entrainment, dispersion (or stirring), and diffusion, spanning a continuous spectrum of space-time scales of the flow [2]. Clearly, turbulent mixing remains a challenging problem in both theoretical and experimental studies.

With growing computational power available nowadays, direct numerical simulation (DNS) has become an indispensable means to study turbulent mixing [3-5]. However, it should be noted that so far most DNS studies of turbulent mixing are limited to that of passive scalars (e.g., $[4,6])$, in which scalar dynamics is driven by, but does not affect hydrodynamics. Theory, modeling, and simulation of realistic mixing flows, which are characterized by active-scalar dynamics of the mixed species and/or chemically reacting components, are not yet well developed and remain as an open and active research area [1]. In active-scalar dynamics, the scalar dynamics affects the mixture hydrodynamics and vice verse, as opposed to passive-scalar dynamics which is driven by, but does not any influence to, hydrodynamics. The example of the former is a binary mixture of two species of different molecular weights, and that of the latter is a mixture of two species of different color but otherwise identical properties.

The demand for effective modeling and simulation strategies is particular pressing for combustion, which includes a broad class of problems, e.g., reacting scalar mixing layers [7], mixing of a conserved scalar in a turbulent reacting flows [8], modeling of both premixed [9] and non-premixed [10] flames, detonations in mixtures [11] and mixing effected by compressibility in high Reynolds number flows. For these problems, the theories and models based on the passive-scalar approach may not be adequate for the reasons which are discussed later (cf. Sec. 3.3). In turbulent mixing, one must deal with the effects due to variable Schmidt number Sc and species of different molecular properties in mixtures, among other things. In continuum theory for mixtures $[12,13]$, one is inevitably confronted with the closure problem of expressing the barycentric mixture dynamics in terms of the individual species dynamics (e.g., $[14,15])$. An example of the closure problem can be illustrated by combining two single-species momentum equations to obtain a single-fluid momentum equation for the multi-species mixture in terms of the barycentric velocity. The resulting mixture momentum equation involves quantities of individual species, which must be approximated by the mixture properties in order to close the equation. This closure problem is difficult to model within the frame- 
work of continuum theory because it involves inter-species interactions, which can only be properly treated by means of statistical mechanics in principle $[14,15]$.

In this work we will develop a lattice Boltzmann equation (LBE) for mixtures with active-scalar mixing dynamics. There already exists a number of lattice Boltzmann models for mixture (e.g., [16,17]). However, most existing LBE models for mixtures have no direct connection to kinetic theory and are based on heuristics $[18,19]$. Only very recently some LBE models for mixtures have been directly derived from kinetic equations [20-23] and this work is a continuation of our effort in this direction. The LBE model for mixtures proposed in this work has several new features. First, it is directly derived from the linearized Boltzmann equations for mixtures. Second, it uses the multiple-relaxation-time collision model [24-28] as opposed to the popular Bhatnagar-Gross-Krook (BGK) model [29] with single-relaxation-time, which has been used in most existing LBE models for multi-component fluids (e.g., $[16,17])$. Finally and most importantly, the proposed model has a consistent baroclinic coupling between the species dynamics and the mixture dynamics and satisfies the indifferentiability principle, both of which have not been adequately addressed in the previous LBE models. The proposed LBE model is for mixtures of ideal gases in near incompressible conditions and will be tested for simulations of scalar dynamics in decaying homogeneous isotropic turbulence (DHIT) in three dimensions.

Decaying homogeneous isotropic turbulence (DHIT) is a canonical test in turbulence theory and is a most studied flow with DNS (cf. [6] and references therein). We note that while DNS studies on DHIT of incompressible flows of single species are extensive (cf. [6]), including recent results obtained by using the LBE method [30,31], there are relatively few on DNS of compressible turbulence [32-35], and even less on DNS study of DHIT in mixtures beyond passive scalar mixing (e.g., $[4,6])$. We hope our present work on DNS of DHIT in mixtures can induce further research interest in this area.

The remainder of this paper is organized as follows. In Sec. 2 we review the kinetic theory and various model kinetic equations for mixtures. In particular, we discuss the importance of the indifferentiability principle in deriving consistent hydrodynamic equations. In Sec. 3 we discuss in detail the proposed lattice Boltzmann equation for mixture in three dimensions, including the hydrodynamic equations, the transport coefficients, and scalar dynamics derived from the proposed LBE model. In Sec. 4 we discuss the numerics of the LBE simulations of mixtures. One particular important aspect for activescalar dynamics in mixtures is initialization of flow fields, which is significantly different from that in passive-scalar dynamics. Because species density and velocity fields must be consistent with each other, we propose an initialization procedure for this purpose. For completeness, we also provide a brief descrip- 
tion of decaying homogeneous isotropic turbulence. In Sec. 5 we show our preliminary results in two parts: the scalar dynamics in mixing and DNS of DHIT. Specifically, we measure the kinetic energy $K(t)$ and the dissipation rate $\varepsilon(t)$ and the decay exponent $n$. When comparing our results with existing data, we find our results agree with previous results. Finally, we conclude the paper in Sec. 6

\section{Kinetic and hydrodynamic theory for mixtures}

We will discuss succinctly the kinetic theory for mixtures. For the sake of simplicity without loss of generality, we shall restrict this discussion to the Boltzmann equations for binary mixtures in what follows. The simultaneous Boltzmann equations for a binary mixture without external force can be written as:

$$
\partial_{t} f^{\sigma}+\boldsymbol{\xi} \cdot \nabla f^{\sigma}=Q^{\sigma \sigma}+Q^{\sigma \varsigma}
$$

where $Q^{\sigma \varsigma}=Q^{\varsigma \sigma}, \varsigma \neq \sigma$, is the cross collision term for two different species $\sigma$ and $\varsigma$. Obviously, for an $N$-component system, there will be $N$ such equations, each containing $N$ collision terms. In general, the collision term is

$$
Q^{\sigma \varsigma}=\int d \boldsymbol{\xi}_{\varsigma} d \Theta d \varepsilon B\left(\Theta,\left\|\boldsymbol{\xi}_{\sigma \varsigma}\right\|\right)\left[f^{\prime \sigma} f^{\prime \varsigma}-f^{\sigma} f^{\varsigma}\right]
$$

where $f^{\prime \sigma}\left(f^{\prime \varsigma}\right)$ and $f^{\sigma}\left(f^{\varsigma}\right)$ denote the post-collision and pre-collision state of the particle of species $\sigma(\varsigma)$, respectively, $\boldsymbol{\xi}_{\sigma \varsigma}:=\left(\boldsymbol{\xi}_{\sigma}-\boldsymbol{\xi}_{\varsigma}\right)$, and we refer the details of the collision integral of Eq. (2) to standard texts on the Boltzmann equation [36-40]. Obviously, the system of $N$ equations for $N$ species is much more formidable to analyze than the Boltzmann equation for a single-species system. In modeling of an $N$-species system, the first objective is to find a suitable approximation for the integral collision term of Eq. (2) that would significantly simplify the computation while maintaining the most essential part of the physics. For this purpose, the linearized or relaxation collision models are applied [39-41].

The justification for the relaxation approximation for the collision terms relies on our understanding of the underlying physics pertinent to mixtures. Consider two binary mixtures, for example, each consisting of a light and a heavy gas. The total mass of each species is equal for one mixture, implying a smaller number density for the heavier gas, and the number densities of the two species is equal for the other, implying a larger mass density (or mass fraction) for the heavier species. In equal-mass mixture, the Maxwellization of light species is mostly due to self-collision whereas the equilibration of the heavier species is predominantly due to cross collisions. This is due to the fact that the number of heavy molecules available for collisions is smaller. In the 
equal-number mixture, Maxwellization of both species involves self and cross collisions. This example illustrates the equilibrating process in a mixture depends strongly on the properties of the mixture. When the Maxwellization process is complete, the stress of the corresponding species becomes isotropic, or equivalently the heat conduction relaxes to zero. Therefore, the scale on which the stress becomes isotropic or the heat conduction relaxes is a suitable measure of Maxwellization. The equilibration among different species can also take place in several different manners. Velocity and temperature differences may equilibrate on the same temporal scale, as in the equal-mass mixture, or on vastly different scales, as in the equal-number mixture. In addition, these equilibrating processes need not occur sequentially but also concurrently with the Maxwellization.

There is a significant amount of literature on gas mixtures within the framework of kinetic theory $[37,41-52]$. In the Chapman-Enskog analysis for a simple gas, one assumes a clear separation of scales in space and time, that is, to distinguish the spatial and temporal scales which are much larger than the mean free path or mean free time, respectively. An analogy for a mixture becomes much more difficult because of multiplicity of spatial and temporal scales due to inter-species interactions. In the work of Chapman and Cowling [37], the full Boltzmann equations for a binary mixture are analyzed under the assumptions that all scales are of the same order approximately, or equivalently, that the phenomenon of interest is smooth with respect to all collisional scales. The determination of various transport coefficients was the main objective of Chapman and Cowling [37] and no attempt was made to describe the evolution dynamics for mixtures.

Direct analysis or numerical simulation of the Boltzmann equation is not feasible in general. This is due to the difficulty involved in evaluating the complex integral collision operators. Two approaches can be followed to circumvent this difficulty. The first, Grad's moment method, is to obtain the non-normal solutions of the Boltzmann equation (i.e., the solutions beyond the hydrodynamic variables) [53]. The Boltzmann equation is equivalent to a system of infinite number of moment equations. In the Grad's moment method, the moment system is truncated to a finite number of moments and closure modeling is required to express the unclosed moments in terms of the closed ones. The second method is to derive simplified model Boltzmann equations which are more manageable to solve. Numerous model equations are influenced by Maxwell's approach to solve the Boltzmann equation by using the properties of the Maxwell molecule [54] and the linearized Boltzmann equation. The simplest model equations for a binary mixture is that by Gross and Krook [43], which is an extension of the single-relaxation-time model for a pure system the celebrated Bhatnagar-Gross-Krook (BGK) model [29].

With the BGK approximation [29,43], the collision integrals $Q^{\sigma \varsigma}[\sigma, \varsigma \in$ 
$\{\mathrm{A}, \mathrm{B}\}]$ can be approximated by following linearized collision terms

$$
J^{\sigma \sigma}=-\frac{1}{\lambda_{\sigma}}\left[f^{\sigma}-f^{\sigma(0)}\right], \quad J^{\sigma \varsigma}=-\frac{1}{\lambda_{\sigma \varsigma}}\left[f^{\sigma}-f^{\sigma \varsigma(0)}\right],
$$

where $f^{\sigma(0)}$ and $f^{\sigma \varsigma(0)}$ are Maxwellians

$$
\begin{aligned}
& f^{\sigma(0)}\left(\rho_{\sigma}, \boldsymbol{u}_{\sigma}, T_{\sigma}\right)=\frac{\rho_{\sigma}}{\left(2 \pi R_{\sigma} T_{\sigma}\right)^{D / 2}} e^{-\left(\boldsymbol{\xi}-\boldsymbol{u}_{\sigma}\right)^{2} /\left(2 R_{\sigma} T_{\sigma}\right)}, \\
& f^{\sigma \varsigma(0)}\left(\rho_{\sigma}, \boldsymbol{u}_{\sigma \varsigma}, T_{\sigma \varsigma}\right)=\frac{\rho_{\sigma}}{\left(2 \pi R_{\sigma} T_{\sigma \varsigma}\right)^{D / 2}} e^{-\left(\boldsymbol{\xi}-\boldsymbol{u}_{\sigma \varsigma}\right)^{2} /\left(2 R_{\sigma} T_{\sigma \varsigma}\right)},
\end{aligned}
$$

where $D$ is the spatial dimension, $R_{\sigma}=k_{B} / m_{\sigma}$ and $m_{\sigma}$ are the gas constant and the molecular mass of the $\sigma$ species, respectively, and $k_{B}$ is the Boltzmann constant. There are three adjustable relaxation parameters in the collision terms: $\lambda_{\sigma}, \lambda_{\varsigma}$, and $\lambda_{\sigma \varsigma}=\left(\rho_{\varsigma} / \rho_{\sigma}\right) \lambda_{\varsigma \sigma}$. The species Maxwellian $f^{\sigma(0)}$ is characterized by the conserved variables of each individual species: the mass density $\rho_{\sigma}$, the mass velocity $\boldsymbol{u}_{\sigma}$, and the temperature $T_{\sigma}$; while the mixture Maxwellians $f^{\sigma \varsigma(0)}$ and $f^{\varsigma \sigma(0)}$ are characterized by four adjustable parameters: $\boldsymbol{u}_{\sigma \varsigma}, \boldsymbol{u}_{\varsigma \sigma}, T_{\sigma \varsigma}$, and $T_{\varsigma \sigma}$. There are several considerations in determining these arbitrary parameters: simplicity of the resulting theory, accuracy of approximation, and ease of computation. The cross-collisional terms would be symmetric only if one takes $\boldsymbol{u}_{\sigma \varsigma}=\boldsymbol{u}_{\varsigma \sigma}=\boldsymbol{u}$ and $T_{\sigma \varsigma}=T_{\varsigma \sigma}=T$, where $\boldsymbol{u}$ and $T$ are the velocity and temperature of the mixture, respectively, which are yet to be defined. This is essential in preserving irreversible thermodynamics, especially the Onsager relation [55]. Another thermodynamic relation that needs to be satisfied is the indifferentiability principle [56], that is, if two species are identical, the system of the mixture equations (1) collapses to the equation of a pure species. Obviously, this is true for the Boltzmann equation, but it does not hold for the BGK-type model equations for mixtures. As we shall see later, the constraints imposed by the indifferentiability principle would also affect the self collision terms.

Since a mixture ultimately relaxes to the equilibrium defined by the mixture variables $\boldsymbol{u}$ ant $T$, it is logical to use $f^{\sigma \varsigma(0)}$ as the equilibrium in the ChapmanEnskog analysis. Fewer terms in the expansion of $f^{\sigma}$ about $f^{\sigma \varsigma(0)}$ would be needed in many cases if one chooses $\boldsymbol{u}_{\sigma \varsigma}=\boldsymbol{u}_{\sigma}$ and $T_{\sigma \varsigma}=T_{\sigma}$, i.e., $f^{\sigma \varsigma(0)}=f^{\sigma(0)}$. The main difference in using the mixture variables $\boldsymbol{u}$ and $T$, as opposed to the species variables $\boldsymbol{u}_{\sigma}$ and $T_{\sigma}$ is that the former leads to the single-fluid theory, from which one set of hydrodynamic equations for the mixture variables is derived, while the latter leads to the two-fluid theory [45,51], from which two sets of coupled hydrodynamic equations of mixture variables can be derived.

For $\sigma$ species, the BGK-type collision term combining self- and cross-collisions 
can be rewritten as:

$$
J_{\sigma}=-\left(\frac{1}{\lambda_{\sigma}}+\frac{1}{\lambda_{\sigma \varsigma}}\right)\left[f^{\sigma}-f^{\sigma(0)}\right]-\frac{1}{\lambda_{\sigma \varsigma}}\left[f^{\sigma(0)}-f^{\sigma \varsigma(0)}\right] .
$$

Mathematically, $f^{\sigma(0)}$ can be expanded in terms of $f^{\sigma \varsigma(0)}$, or equivalently, the fluid properties of individual species, $\rho_{\sigma}, \boldsymbol{u}_{\sigma}$, and $T_{\sigma}$ in terms of the mixture fluid properties, $\rho, \boldsymbol{u}$, and $T$, or vice versa. As pointed out by Gross and Krook [43], and similarly by Hamel $[47,49]$, one can also linearly combine these two expansions with an adjustable parameter $0 \leq \beta \leq 1$, that is, a portion of $f^{\sigma(0)}$, $\beta f^{\sigma(0)}$, is expressed in terms of $f^{\sigma \varsigma(0)}$, and a portion of $f^{\sigma \varsigma(0)},(1-\beta) f^{\sigma \varsigma(0)}$, is expressed in terms $f^{\sigma(0)}$ :

$$
\begin{aligned}
& f^{\sigma(0)}-f^{\sigma \varsigma(0)}=n_{\sigma}\left(2 \pi R_{\sigma} T\right)^{-3 / 2}\left(e^{-c_{\sigma}^{2} / 2 R_{\sigma} T}-e^{-c^{2} / 2 R_{\sigma} T}\right) \\
= & (1-\beta) f^{\sigma(0)}\left(1-e^{-\left(2 \boldsymbol{c}_{\sigma}+\boldsymbol{w}_{\sigma}\right) \cdot \boldsymbol{w}_{\sigma} / 2 R_{\sigma} T}\right) \\
& -\beta f^{\sigma \varsigma(0)}\left(1-e^{\left(2 \boldsymbol{c}-\boldsymbol{w}_{\sigma}\right) \cdot \boldsymbol{w}_{\sigma} / 2 R_{\sigma} T}\right),
\end{aligned}
$$

where $\boldsymbol{c}:=(\boldsymbol{\xi}-\boldsymbol{u}), \boldsymbol{c}_{\sigma}:=\left(\boldsymbol{\xi}-\boldsymbol{u}_{\sigma}\right), \boldsymbol{w}_{\sigma}:=\left(\boldsymbol{u}_{\sigma}-\boldsymbol{u}\right)$, and we have assumed the mixture is isothermal, i.e., $T_{\sigma}=T_{\sigma \varsigma}=T$.

If the cross-collision term is linearized in terms of the diffusion velocity $\boldsymbol{w}_{\sigma}:=$ $\left(\boldsymbol{u}_{\sigma}-\boldsymbol{u}\right)$, one obtains the generalized model of Sirovich [45]:

$$
J_{\sigma}=-\frac{1}{\lambda_{\sigma}}\left[f^{\sigma}-f^{\sigma(0)}\right]-\frac{1}{\lambda_{\sigma \varsigma}} \boldsymbol{s}_{\sigma} \cdot \boldsymbol{w}_{\sigma}
$$

where

$$
\boldsymbol{s}_{\sigma}:=\frac{1}{R_{\sigma} T}\left[(1-\beta) f^{\sigma(0)}\left(\boldsymbol{\xi}-\boldsymbol{u}_{\sigma}\right)+\beta f^{\sigma \varsigma(0)}(\boldsymbol{\xi}-\boldsymbol{u})\right] .
$$

The original model of Sirovich [45] is recovered when $\beta=0$. This model allows two relaxation times, consequently an independent variable Schmidt number Sc.

So far we have yet to define the mixture velocity $\boldsymbol{u}$ and temperature $T$. The choice of $\boldsymbol{u}$ and $T$ is unique and is a key issue in the BGK-type of modeling. By insisting that the relaxation equations for the velocity difference $\left(\boldsymbol{u}_{\sigma}-\boldsymbol{u}_{\varsigma}\right)$ and the temperature difference $\left(T_{\sigma}-T_{\varsigma}\right)$ obtained from the full Boltzmann equations and the model equations must be the same, the following definitions for the mixture velocity and temperature must be used [46]:

$$
\begin{aligned}
& \boldsymbol{u}=\boldsymbol{u}_{\sigma \varsigma}=\boldsymbol{u}_{\varsigma \sigma}=\frac{m_{\sigma} \boldsymbol{u}_{\sigma}+m_{\varsigma} \boldsymbol{u}_{\varsigma}}{m_{\sigma}+m_{\varsigma}} \\
& T_{\sigma \varsigma}=T_{\sigma}+\frac{2 m_{\sigma} m_{\varsigma}}{\left(m_{\sigma}+m_{\varsigma}\right)^{2}}\left[\left(T_{\varsigma}-T_{\sigma}\right)+\frac{m_{\varsigma}}{6 k_{B}}\left(\boldsymbol{u}_{\varsigma}-\boldsymbol{u}_{\sigma}\right)^{2}\right] .
\end{aligned}
$$


However, the above definition of $\boldsymbol{u}$ for the BGK model equations contradicts the indifferentiability principle [56]. That is, for two identical species $\sigma$ and $\varsigma$, the model equations do not reduce to the one for a single species gas. For the BGK model equations of mixtures, the indifferentiability principle can be maintained if the barycentric velocity is used in the mixture Maxwellian:

$$
\boldsymbol{u}=\frac{\rho_{\sigma} \boldsymbol{u}_{\sigma}+\rho_{\varsigma} \boldsymbol{u}_{\varsigma}}{\rho_{\sigma}+\rho_{\varsigma}}
$$

But the barycentric velocity is inconsistent with the conditions (9a) derived from the full Boltzmann equations. Hence, a dilemma arises since a choice has to be made between satisfying either the full Boltzmann equation or the indifferentiability principle. As we will argue next, the indifferentiability principle is more important for the hydrodynamic modeling of mixtures considered here.

\section{A lattice Boltzmann model for mixture and its hydrodynamics}

\subsection{The lattice Boltzmann equations}

In the previous section, the distinction between self and cross collisions is highlighted, as it should be when discussing different physical regimes. Some practical examples allow the reader to understand the role of these collisions in approaching equilibrium by different mixtures. Modeling these interactions by means of two split collisional operators seems the most natural choice. However two doubts immediately arise: first of all, in case of many species, the cross collision term describes many different interactions at the same time; secondly, and more seriously, in case of linear BGK-type models, the model splitting in two operators can be easily overcome by regrouping them in one global collisional operator, with a new redefined equilibrium [23]. Hence the distinction between the split collisional operators and the global one is simply a matter of defining a consistent local equilibrium. Clearly for this second goal, the Indifferentiability Principle should be taken into account. This has already be recognized in the recent kinetic theory of mixtures [56].

The lattice Boltzmann equations for a binary mixture can be written as the following:

$$
\mathbf{f}^{\sigma}\left(\boldsymbol{x}_{j}+\boldsymbol{c} \delta t, t+\delta t\right)-\mathbf{f}^{\sigma}\left(\boldsymbol{x}_{j}, t\right)=-\mathbf{M}^{-1} \cdot \hat{\mathrm{S}} \cdot \mathbf{M} \cdot\left[\mathbf{f}^{\sigma}-\mathbf{f}^{\sigma(\mathrm{eq})}(\boldsymbol{u})\right]
$$

where $\boldsymbol{u}$ is the barycentric velocity of the mixture, the fold-face symbols such as $\mathbf{f}$ denote $Q$-tuple vectors, and $Q$ is the number of discrete velocities: 


$$
\begin{aligned}
& \mathbf{f}^{\sigma}:=\left(f_{0}^{\sigma}, f_{1}^{\sigma}, \ldots, f_{N}^{\sigma}\right)^{\top}, \\
& \mathbf{f}^{\sigma(\mathrm{eq})}:=\left(f_{0}^{\sigma(\mathrm{eq})}, f_{1}^{\sigma(\mathrm{eq})}, \ldots, f_{N}^{\sigma(\mathrm{eq})}\right)^{\top}, \\
& \mathbf{f}^{\sigma}\left(\boldsymbol{x}_{j}+\boldsymbol{c} \delta t\right):=\left(f_{0}^{\sigma}\left(\boldsymbol{x}_{j}\right), f_{1}^{\sigma}\left(\boldsymbol{x}_{j}+\boldsymbol{c}_{1} \delta t\right), \ldots, f_{N}^{\sigma}\left(\boldsymbol{x}_{j}+\boldsymbol{c}_{N} \delta t\right)\right)^{\top},
\end{aligned}
$$

where $T$ denotes the transpose operator. In Eq. (11), the equilibrium distribution functions are given by:

$$
f_{i}^{\sigma(\mathrm{eq})}=w_{i}\left\{\alpha_{\sigma i} \rho_{\sigma}+\frac{\left(\boldsymbol{c}_{i} \cdot \boldsymbol{u}\right)}{R_{\sigma} T}+\frac{1}{2}\left[\frac{\left(\boldsymbol{c}_{i} \cdot \boldsymbol{u}\right)^{2}}{\left(R_{\sigma} T\right)^{2}}-\frac{\boldsymbol{u} \cdot \boldsymbol{u}}{R_{\sigma} T}\right]\right\} .
$$

It should be noted that collision term in the LBE model of Eq. (11) has indeed combined both self and mutual collision terms, because the equilibrium $f_{i}^{\sigma(\mathrm{eq})}$ is a function of the barycentric velocity $\boldsymbol{u}$. Therefore, interactions between the species have been considered in the collision term.

We will use 19 velocity model in three dimensions (D3Q19 model), of which the discrete velocities are: $\boldsymbol{c}_{0}=(0,0,0), \boldsymbol{c}_{i}=( \pm 1,0,0) c,(0, \pm 1,0) c$, and $(0,0, \pm 1) c$, for $i=1-6$, and $\boldsymbol{c}_{i}=( \pm 1, \pm 1,0) c,( \pm 1,0, \pm 1) c$, and $(0, \pm 1, \pm 1) c$, for $i=7-18$, where $c:=\delta_{x} / \delta_{t}$. For this model, the coefficients $w_{0}=1 / 3$, $w_{i}=1 / 18$ for $\left\|\boldsymbol{c}_{i}\right\|=1, i=1-6$, and $w_{i}=1 / 36$ for $\left\|\boldsymbol{c}_{i}\right\|=\sqrt{2}, i=7-18$; the parameter $R_{\sigma} T=1 / 3$ in the model; and $\alpha_{\sigma 0}=\left(3-2 \varphi_{\sigma}\right)$ and $\alpha_{\sigma i}=\varphi_{\sigma}$ for $i \neq 0$, and $0<\varphi_{\sigma} \leq 1$ is an adjustable parameter to determine the equation of state for $\sigma$-species:

$$
p_{\sigma}=\frac{1}{3} \varphi_{\sigma} \rho_{\sigma}
$$

With a specific order of the moments as the following:

$$
\mathbf{m}:=\left(\rho, u_{x}, u_{y}, u_{z}, p_{x y}, p_{x z}, p_{y z}, 3 p_{x x}, p_{w w}, e, q_{x}, m_{x}, q_{y}, m_{y}, q_{z}, m_{z}, e_{1}, e_{2}, e_{3}\right)^{\top},
$$

the transform matrix $\mathrm{M}$ is given by:

$$
\mathbf{M}=\left(\begin{array}{ccccccccccccccccccc}
1 & 1 & 1 & 1 & 1 & 1 & 1 & 1 & 1 & 1 & 1 & 1 & 1 & 1 & 1 & 1 & 1 & 1 & 1 \\
0 & 1 & -1 & 0 & 0 & 0 & 0 & 1 & -1 & 1 & -1 & 1 & -1 & 1 & -1 & 0 & 0 & 0 & 0 \\
0 & 0 & 0 & 1 & -1 & 0 & 0 & 1 & 1 & -1 & -1 & 0 & 0 & 0 & 0 & 1 & -1 & 1 & -1 \\
0 & 0 & 0 & 0 & 0 & 1 & -1 & 0 & 0 & 0 & 1 & 1 & -1 & -1 & 1 & 1 & -1 & -1 \\
0 & 0 & 0 & 0 & 0 & 0 & 0 & 1 & -1 & -1 & 1 & 0 & 0 & 0 & 0 & 0 & 0 & 0 & 0 \\
0 & 0 & 0 & 0 & 0 & 0 & 0 & 0 & 0 & 0 & 0 & 1 & -1 & -1 & 1 & 0 & 0 & 0 & 0 \\
0 & 0 & 0 & 0 & 0 & 0 & 0 & 0 & 0 & 0 & 0 & 0 & 0 & 0 & 0 & 1 & -1 & -1 & 1 \\
0 & 2 & 2 & -1 & -1 & -1 & -1 & 1 & 1 & 1 & 1 & 1 & 1 & 1 & 1 & -2 & -2 & -2 & -2 \\
0 & 0 & 0 & 1 & 1 & -1 & -1 & 1 & 1 & 1 & 1 & -1 & -1 & -1 & -1 & 0 & 0 & 0 & 0 \\
-30 & -11 & -11 & -11 & -11 & -11 & -11 & 8 & 8 & 8 & 8 & 8 & 8 & 8 & 8 & 8 & 8 & 8 & 8 \\
0 & -1 & 1 & 0 & 0 & 0 & 0 & 2 & -2 & 2 & -2 & -1 & 1 & -1 & 1 & 0 & 0 & 0 & 0 \\
0 & -1 & 1 & 0 & 0 & 0 & 0 & 0 & 0 & 0 & 0 & 1 & -1 & 1 & -1 & 0 & 0 & 0 & 0 \\
0 & 0 & 0 & -1 & 1 & 0 & 0 & 2 & 2 & -2 & -2 & 0 & 0 & 0 & 0 & -1 & 1 & -1 & 1 \\
0 & 0 & 0 & -1 & 1 & 0 & 0 & 0 & 0 & 0 & 0 & 0 & 0 & 0 & 0 & 1 & -1 & 1 & -1 \\
0 & 0 & 0 & 0 & 0 & -1 & 1 & 0 & 0 & 0 & 0 & 2 & 2 & -2 & -2 & -1 & -1 & 1 & 1 \\
0 & 0 & 0 & 0 & 0 & -1 & 1 & 0 & 0 & 0 & 0 & 0 & 0 & 0 & 0 & 1 & 1 & -1 & -1 \\
1 & -5 & -5 & -5 & -5 & 4 & 4 & 7 & 7 & 7 & 7 & -2 & -2 & -2 & -2 & -2 & -2 & -2 & -2 \\
1 & -5 & -5 & 2 & 2 & -3 & -3 & 0 & 0 & 0 & 0 & 5 & 5 & 5 & 5 & -2 & -2 & -2 & -2 \\
1 & 0 & 0 & -3 & -3 & -3 & -3 & 0 & 0 & 0 & 0 & 0 & 0 & 0 & 0 & 3 & 3 & 3 & 3
\end{array}\right)
$$

The matrix $\mathrm{M}$ maps the distribution function to its moments:

$$
\mathbf{m}=\mathrm{M} \cdot \mathbf{f}, \quad \mathbf{f}=\mathrm{M}^{-1} \cdot \mathbf{m} .
$$


The labeling of the discrete velocity set $\left\{\boldsymbol{c}_{i}\right\}$ is uniquely defined by the rows 2, 3 and 4 in $\mathrm{M}$ corresponding to $u_{x}, u_{y}$ and $u_{z}$, respectively. We note that moments $e_{1}, e_{2}$ and $e_{3}$ and linear combinations of moments $\varepsilon, \pi_{x x}$ and $\pi_{w w}$ in [27]. For the construction of $\mathrm{M}$ and detailed description of the moments, we refer readers to the work by d'Humières et al. [27].

The diagonal matrix $\hat{\mathrm{S}}$ of relaxation rates $\left\{s_{i}\right\}$ is given by:

$$
\hat{\mathrm{S}}=\operatorname{diag}\left(s_{1}, s_{D}, s_{D}, s_{D}, s_{\nu}, s_{\nu}, s_{\nu}, s_{\nu}, s_{\nu}, s_{\zeta}, s_{11}, \ldots, s_{19}\right)
$$

Because $\boldsymbol{u}_{\sigma}=\left(m_{1}, m_{2}, m_{3}\right)=\left(u_{\sigma x}, u_{\sigma y}, u_{\sigma z}\right)$ and $\boldsymbol{u}=\left(m_{1}^{(\mathrm{eq})}, m_{2}^{(\mathrm{eq})}, m_{3}^{(\mathrm{eq})}\right)=$ $\left(u_{x}, u_{y}, u_{z}\right)$, therefore the species velocity $\boldsymbol{u}_{\sigma}$ is relaxed to the mixture velocity $\boldsymbol{u}$, given by the equilibria in the present model. As we shall discuss in the next section, $\left(\boldsymbol{u}_{\sigma}-\boldsymbol{u}\right)$ is related to the diffusion force, thus the relaxation of $\left(\boldsymbol{u}_{\sigma}-\boldsymbol{u}\right)$ models the diffusion process in the system.

\subsection{Hydrodynamic equations}

We use the asymptotic analysis with the diffusive scaling $\delta_{x} / L=O(\epsilon)$ and $\delta_{t} / T=O\left(\epsilon^{2}\right)$ to derive the Navier-Stokes equations from the lattice Boltzmann model, where $L$ and $T$ are typical macroscopic length and time scales. This implies that the macroscopic velocity $U=L / T=\epsilon c$, where $c:=\delta_{x} / \delta_{t}$. The diffusive scaling is appropriate for the incompressible Navier-Stokes equations considered here.

The hydrodynamic equations for $\sigma$-species derived from the LBE model are:

$$
\begin{aligned}
& \partial_{t} \rho_{\sigma}+\nabla \cdot\left(\rho_{\sigma} \boldsymbol{u}_{\sigma}\right)=0, \\
& \partial_{t}\left(\rho_{\sigma} \boldsymbol{u}_{\sigma}\right)+\boldsymbol{u} \cdot \boldsymbol{\nabla}\left(\rho_{\sigma} \boldsymbol{u}\right)=-\nabla p_{\sigma}+\nabla \cdot \mathrm{S}^{\sigma}-s_{D} \rho_{\sigma} \boldsymbol{w}_{\sigma},
\end{aligned}
$$

where $\boldsymbol{w}_{\sigma}:=\left(\boldsymbol{u}_{\sigma}-\boldsymbol{u}\right)=\rho_{\varsigma}\left(\boldsymbol{u}_{\sigma}-\boldsymbol{u}_{\varsigma}\right) / \rho$, the pressure $p_{\sigma}$ is given by Eq. (13), and the stress tensor for the $\sigma$ species is:

$$
\mathrm{S}^{\sigma}=\nu\left[\boldsymbol{\nabla}\left(\rho_{\sigma} \boldsymbol{u}_{\sigma}\right)+\boldsymbol{\nabla}\left(\rho_{\sigma} \boldsymbol{u}_{\sigma}\right)^{\top}-\frac{2}{3} \boldsymbol{\nabla} \cdot\left(\rho_{\sigma} \boldsymbol{u}_{\sigma}\right) \mathrm{I}\right]+\zeta_{\sigma} \boldsymbol{\nabla} \cdot\left(\rho_{\sigma} \boldsymbol{u}_{\sigma}\right) \mathbf{I}
$$

where $\mathrm{T}$ and $\mathrm{I}$ are transpose and identity operators, respectively. The shear viscosity $\nu$ and the bulk viscosity $\zeta_{\sigma}$ are given by:

$$
\begin{aligned}
& \nu=\frac{1}{3}\left(\frac{1}{s_{\nu}}-\frac{1}{2}\right) c \delta_{x}, \\
& \zeta_{\sigma}=\frac{\left(5-3 \varphi_{\sigma}\right)}{9}\left(\frac{1}{s_{\zeta}}-\frac{1}{2}\right) c \delta_{x} .
\end{aligned}
$$


In this work, the relaxation rate, $s_{\nu}$, corresponding to the shear viscosity $\nu$ for each species is equal, therefore the shear viscosity is equal in both species. The bulk viscosity $\zeta_{\sigma}$ depends on both the relaxation rate $s_{\zeta}$ and $\varphi_{\sigma}$, and it is not equal in different species in general. In the present work, we adjust $s_{\zeta}$ so that $\zeta_{\sigma}$ are equal for all species with different $\varphi_{\sigma}$.

The hydrodynamic equations for the mixture barycentric velocity $\boldsymbol{u}$ are:

$$
\begin{aligned}
& \partial_{t} \rho+\nabla \cdot(\rho \boldsymbol{u})=0, \\
& \partial_{t} \rho \boldsymbol{u}+\boldsymbol{u} \cdot \boldsymbol{\nabla}(\rho \boldsymbol{u})=-\nabla p+\nabla \cdot \mathbf{S}
\end{aligned}
$$

where the mixture stress is

$$
\mathrm{S}=\sum_{\sigma} \mathrm{S}^{\sigma}=\nu\left[\boldsymbol{\nabla}(\rho \boldsymbol{u})+\boldsymbol{\nabla}(\rho \boldsymbol{u})^{\top}-\frac{2}{3} \boldsymbol{\nabla} \cdot(\rho \boldsymbol{u}) \mathbf{I}\right]+\sum_{\sigma} \zeta_{\sigma} \boldsymbol{\nabla} \cdot\left(\rho_{\sigma} \boldsymbol{u}_{\sigma}\right) \mathrm{I}
$$

In the low Mach number region, we can assume that $\boldsymbol{\nabla} \cdot \boldsymbol{u} \approx 0$ and the total density gradients $\nabla \rho$ are negligible, then

$$
\mathrm{S}=\sum_{\sigma} \mathrm{S}^{\sigma} \approx \nu \rho\left[(\boldsymbol{\nabla} \boldsymbol{u})+(\boldsymbol{\nabla} \boldsymbol{u})^{\top}\right]
$$

The total pressure of the mixture is

$$
p=\sum_{\sigma} p_{\sigma}=\frac{1}{3} \rho \sum_{\sigma} x_{\sigma} \varphi_{\sigma}
$$

where $x_{\sigma}:=\rho_{\sigma} / \rho$, and the speeds of sound for individual species and mixture are:

$$
c_{s \sigma}^{2}=\frac{1}{3} \varphi_{\sigma} c^{2}, \quad c_{s}^{2}=\frac{1}{3} \varphi c^{2}, \quad \varphi:=\sum_{\sigma} x_{\sigma} \varphi_{\sigma},
$$

where $c:=\delta_{x} / \delta_{t}$.

\subsection{Active and passive scalar dynamics}

The difference of the species momentum equations (17b) leads to the following equation:

$$
\rho_{\sigma}\left(\boldsymbol{u}_{\sigma}-\boldsymbol{u}\right)=-\frac{1}{s_{D}} \nabla p_{\sigma}=-\frac{\varphi_{\sigma} c^{2}}{3 s_{D}} \nabla \rho_{\sigma}=-D_{\sigma} \nabla \rho_{\sigma}
$$

where the species self diffusivity $D_{\sigma}$ is given by:

$$
D_{\sigma}=\frac{\varphi_{\sigma} c^{2}}{3 s_{D}}
$$


Even when the relaxation frequency $s_{D}$ is fixed to be equal for all species, $D_{\sigma}$ can be adjusted by the parameter $\varphi_{\sigma}$. By taking the difference of Eq. (25) for two difference species, we have

$$
s_{D}\left(\boldsymbol{u}_{\sigma}-\boldsymbol{u}_{\varsigma}\right)=-\frac{\rho p}{\rho_{\sigma} \rho_{\varsigma}} \boldsymbol{d}_{\sigma}
$$

where the diffusion force $\boldsymbol{d}_{\sigma}$ is:

$$
\boldsymbol{d}_{\sigma}=\nabla\left(\frac{n_{\sigma}}{n}\right)+\frac{n_{\sigma} n_{\varsigma}}{n \rho}\left(m_{\varsigma}-m_{\sigma}\right) \nabla \ln p .
$$

Therefore, the species mass conservation equation (17a) can be rewritten as:

$$
\partial_{t} \rho_{\sigma}+\boldsymbol{u} \cdot \nabla \rho_{\sigma}=\frac{1}{s_{D}} \nabla \cdot p \boldsymbol{d}_{\sigma}
$$

where we have assumed that $\boldsymbol{\nabla} \cdot \boldsymbol{u}=0$, which is satisfied up to $O\left(\epsilon^{2}\right)$. If we further assume that $\rho, n$ and $p$ for the mixture are approximately constants so that their gradients are negligible, then in the leading order, $\boldsymbol{d}_{\sigma} \approx \boldsymbol{\nabla} n_{\sigma}$, and the above equation (29) is reduced to the advection-diffusion equation for $\rho_{\sigma}$ :

$$
\partial_{t} \rho_{\sigma}+\boldsymbol{u} \cdot \nabla \rho_{\sigma}=-\frac{p}{n m_{\sigma} s_{D}} \nabla^{2} \rho_{\sigma} .
$$

The mutual diffusivity $D_{\sigma \varsigma}$ is defined by the following relation:

$$
\left(\boldsymbol{u}_{\sigma}-\boldsymbol{u}_{\varsigma}\right)=-\frac{n^{2}}{n_{\sigma} n_{\varsigma}} D_{\sigma \varsigma} \boldsymbol{d}_{\sigma \varsigma} .
$$

Consequently, the mutual diffusion coefficient is given by:

$$
D_{\sigma \varsigma}=\frac{p \rho}{s_{D} n^{2} m_{\sigma} m_{\varsigma}}=\frac{m_{\sigma} m_{\varsigma}}{\left(x_{\sigma} m_{\varsigma}+x_{\varsigma} m_{\sigma}\right)^{2}} \sum_{\sigma} x_{\sigma} D_{\sigma},
$$

where $D_{\sigma}$ is the self-diffusion coefficient given by Eq. (26). Therefore, the relaxation rate $s_{D}$ can be used to adjust the mutual diffusion coefficient $D_{\sigma \varsigma}$.

The Navier-Stokes equation (20b) can be re-written as:

$$
\begin{aligned}
& \partial_{t} \boldsymbol{u}+\boldsymbol{u} \cdot \boldsymbol{\nabla} \boldsymbol{u}=-\frac{\varphi c^{2}}{3 \rho} \nabla \rho+\nu \nabla^{2} \boldsymbol{u}+\boldsymbol{F}_{\varphi}+\boldsymbol{F}_{\nu} \\
& \boldsymbol{F}_{\varphi}:=-\frac{c^{2}}{3} \nabla \varphi, \quad \boldsymbol{F}_{\nu}:=\nabla \boldsymbol{u} \cdot \nabla \nu
\end{aligned}
$$

The forces $\boldsymbol{F}_{\varphi}$ and $\boldsymbol{F}_{\nu}$ are unique to the mixture dynamics, the former is due to the spatial inhomogeneity of the mixture speed of sound $c_{s}(\varphi)$ and the latter to the spatial variation of the kinematic viscosity $\nu=\nu(\varphi)$, neither of these forces exists in the single species dynamics. The force $\boldsymbol{F}_{\varphi}$ is the baroclinic 
coupling force which is generated by the misalignments between the pressure and density gradients. The force $\boldsymbol{F}_{\nu}$ is the viscous coupling force. We focus our attention on the effect of $\boldsymbol{F}_{\varphi}$ in the present study and will neglect the effect of $\boldsymbol{F}_{\nu}$, because the viscosity $\nu$ given by Eq. (19a) is assumed to be a constant in the present model by neglecting the effect of local concentration gradients on the mixture viscosity.

One quantity of interest in the mixture dynamics is the order parameter of the mass concentration difference:

$$
\phi:=\frac{\rho_{\sigma}-\rho_{\varsigma}}{\rho_{\sigma}+\rho_{\varsigma}}=\frac{\rho_{\sigma}-\rho_{\varsigma}}{\rho}=x_{\sigma}-x_{\varsigma}
$$

which satisfies the following equation:

$$
\partial_{t} \phi+\boldsymbol{u} \cdot \boldsymbol{\nabla} \phi=\frac{1}{\rho} \boldsymbol{\nabla} \cdot \rho D \nabla \phi+\frac{1}{\rho} \boldsymbol{\nabla} \cdot(\psi-\phi) \nabla p
$$

where $\psi$ is the order parameter of the particle number concentration difference:

$$
\psi:=\frac{n_{\sigma}-n_{\varsigma}}{n_{\sigma}+n_{\varsigma}}=\frac{n_{\sigma}-n_{\varsigma}}{n}=y_{\sigma}-y_{\varsigma} .
$$

For a binary mixture with two equal-mass species, i.e., $m_{\sigma}=m_{\varsigma}$, and constant total density $\rho$, then Eq. (35) reduces to a diffusion equation:

$$
\partial_{t} \phi+\boldsymbol{u} \cdot \nabla \phi=D \nabla^{2} \phi
$$

Even for this simple case, the mass concentration difference $\phi$ in the LBE model is not a passive scalar. In fact the dynamics of $\phi$ influences that of $\varphi:=\sum_{\sigma} x_{\sigma} \varphi_{\sigma}$, which results in baroclinic coupling force $\boldsymbol{F}_{\varphi}:=-c^{2} \boldsymbol{\nabla} \varphi / 3$. The baroclinic coupling force $\boldsymbol{F}_{\varphi}$ in turn drives the mixture hydrodynamics, as indicated in Eq. (33a), while $\phi$ is also driven by the hydrodynamics. Consequently, the scalar dynamics of $\phi$ is closely coupled with the mixture hydrodynamics, as opposed to the passive-scalar case, in which $\phi$ is passively driven by the hydrodynamics, and has no effect on the hydrodynamics $[4,6]$. Therefore, the order parameter $\phi$ is generally an active scalar, which would become a passive scalar only when $m_{\sigma}=m_{\varsigma}$ and $\varphi_{\sigma}=\varphi_{\varsigma}$, provided that all other parameters, such as the relaxation rates $s_{i}$, are identical for both species. Under such conditions, two species are identical, only if the indifferentiability principle is satisfied. The above analysis also indicates how the viscous coupling force $\boldsymbol{F}_{\nu}$ can be introduced in the mixture by the dependence of the mixture viscosity $\nu$ on the local species concentrations $x_{\sigma}$.

While $\phi$ is an active scalar in reality, the proposed LBE model can simulate the dynamics of a passive scalar by treating $\phi$ as a constant $\phi_{0}$ in Eq. (33a). In doing so, the barycentric velocity $\boldsymbol{u}$ affects the species dynamics, but not 
vice versa. The implementation of passive scalar simulations will be further discussed in Sec. 4.1.

\section{Numerics in Simulations}

Several programming practices should be adopted in LBE codes to enhance computational speed and save memory. First, one must not use 2D arrays for the transform matrix $M$ in coding, because because calculations involving arrays of different dimensions are very inefficient. Therefore, when computing moments from the distributions and vice verse, one should explicitly carry out the calculations. Second, one should eliminate common sub-expressions by grouping them in brackets. This would take advantage of compiler to reduce the number of floating point operations (FLOP). Thirdly, one should code the entire collision step together, and not code calculations of moments separately as a subroutine. In addition, if possible, one should combine collision and advection together to reduce memory access time. With these sound commonsense practices, an MRT-LBE code is about $15 \%$ slower than the LBGK code with equal number of discrete velocities. However, the MRT-LBE model is far more efficient because of its numerical stability and flexibility.

For 3D calculations, parallel computing is necessary. Our code is written in $\mathrm{C}++$ with a version of open source Message Passing Interface library (MPICH 1.3). For the discrete fast Fourier transform, we use open source library FFTW. The 3D simulations presented in this work were carried out on cluster computers available to us at the Department of Computer Science, Old Dominion University (ODU) and Politecnico di Torino.

\subsection{Initial conditions}

To carry out the simulation of decaying homogeneous isotropic turbulence in a binary mixture, correct initial conditions are crucial. It is important to stress that even though we are interested in the incompressible flows in the present study, the mixture does introduce compressibility. This makes the initialization for mixture fluids of mixtures more complicated than single-species fluids. We will first discuss the initialization procedure consistent with the active scalar methodology. We will also discuss the initialization procedure for passive scalar methodology adopted for the LBE method.

In simulations of decaying homogeneous isotropic turbulence, an initial energy

spectrum in the Fourier space $\boldsymbol{k}$ is given, $\tilde{E}_{0}(k):=\tilde{E}(k, t=0)$ for $k=\|\boldsymbol{k}\|$. For mixtures, it is the individual species momentum $\boldsymbol{j}_{\sigma}$, rather than the individual 
species velocity $\boldsymbol{u}_{\sigma}$, which has to satisfy the divergence free condition, i.e., $\boldsymbol{\nabla} \cdot \boldsymbol{j}_{\sigma}=0$. We therefore use the following initialization procedure for mixture flows:

(1) A random momentum for $\sigma$-species, $\boldsymbol{j}_{\sigma}:=\rho_{\sigma} \boldsymbol{u}_{\sigma}$, is generated in the physical space $\boldsymbol{x}$ first and then is made divergence free in the Fourier space $\boldsymbol{k}$ so that $\boldsymbol{k} \cdot \tilde{\boldsymbol{j}}_{\sigma}=0$.

(2) Set $\rho_{\sigma}=1$ initially, therefore $\tilde{\boldsymbol{j}}_{\sigma} \cdot \tilde{\boldsymbol{\jmath}}_{\sigma} / 2=\tilde{E}_{0}(k)$. Adjust the magnitude of $\tilde{\boldsymbol{j}}_{\sigma}(k)$ according to $\tilde{E}_{0}(k)$. Now $\boldsymbol{j}_{\sigma}(\boldsymbol{x})$ is divergence free and satisfies the specified initial energy spectrum $\tilde{E}_{0}(k)$.

(3) With $\boldsymbol{j}_{\sigma}(\boldsymbol{x})$ given, we generate consistent density field $\rho_{\sigma}$ and velocity field $\boldsymbol{u}_{\sigma}$ through an iterative procedure. We initialize the density field $\rho_{\sigma}^{[0]}=1$, then $\boldsymbol{u}_{\sigma}^{[0]}=\boldsymbol{j}_{\sigma}$, which are used as initial conditions for a singlespecies lattice Boltzmann equation. After one collision-advection cycle, the single-species LBE generates a new $\rho_{\sigma}^{[1]}$ and $\boldsymbol{u}_{\sigma}^{[1]}=\boldsymbol{j}_{\sigma} / \rho_{\sigma}^{[1]}$. With $\boldsymbol{j}_{\sigma}$ fixed, this process is iterated until steady solutions of $\rho_{\sigma}=\lim _{n \rightarrow \infty} \rho_{\sigma}^{[n]}$ and $\boldsymbol{u}_{\sigma}=\boldsymbol{j} / \rho_{\sigma}$ are obtained by setting a criterion $\left\|\rho_{\sigma}^{[n+1]}-\rho_{\sigma}^{[n]}\right\| \leq \epsilon$ (or $\left.\left\|\boldsymbol{u}_{\sigma}^{[n+1]}-\boldsymbol{u}_{\sigma}^{[n]}\right\| \leq \epsilon\right)$, with some small $\epsilon>0$.

(4) The iterative procedure is repeated for each species.

With the momentum, density and velocity fields generated consistently with each other for a specified initial energy spectrum $\tilde{E}_{0}(k)$ for each species, the mixture momentum $\boldsymbol{j}:=\sum_{\sigma} \boldsymbol{j}_{\sigma}$ is of course divergence free and satisfies the initial energy spectrum $\tilde{E}_{0}(k)$.

Since most existing studies of turbulent mixing is concerned with passive scalars [4], we must compare our method with the passive-scalar approach. The initialization for passive scalar mixing is rather different from that for active scalar mixing. For completeness, we shall describe succinctly the initialization procedure [4] as follows.

(1) For $\sigma$-species, the initial density $\rho_{\sigma}(\boldsymbol{x}, 0)$ is first generated in the Fourier space $\boldsymbol{k}$ :

$$
\tilde{\rho}_{\sigma}(\boldsymbol{k}, 0)=\sqrt{\frac{f_{\rho}(k)}{4 \pi k^{2}}} e^{i 2 \pi \theta(\boldsymbol{k})},
$$

where $\theta(\boldsymbol{k})$ is uniformly distributed random number between 0 and 1 , and $f_{\rho}(k)$ is a "top-hat" function:

$$
f_{\rho}(k)=\left\{\begin{array}{l}
1,\left|k-k_{s}\right| \leq k_{0} / 2 \\
0,\left|k-k_{s}\right|>k_{0} / 2
\end{array}\right.
$$

The ratio $k_{0} / k_{s}$ basically determines the integral length scale of the scalar field of $\rho_{\sigma}$; larger values of $k_{0} / k_{s}$ lead to larger length scales for the density field $\rho_{\sigma}[4]$. 
(2) The density $\rho_{\sigma}(\boldsymbol{x}, 0)$ in physical space $\boldsymbol{x}$ is obtained by inverse-Fourier transform of $\tilde{\rho}_{\sigma}(\boldsymbol{k}, 0)$. In physical space $\rho_{\sigma}(\boldsymbol{x}, 0)$ at each node is reset to $\rho_{\sigma}^{\min }$, if it is positive, and to $\rho_{\sigma}^{\max }$, if it is negative.

(3) The "double-delta" density distribution function created in the previous step has abruptive variations from one node to next in physical space, which are not resolved properly and therefore need to be smoothed. Thus the "double-delta" density distribution function is transferred to $\boldsymbol{k}$-space and its Fourier amplitudes are multiplied by the following filter function:

$$
F_{\rho}(k)= \begin{cases}1, & k \leq k_{c} \\ \left(k / k_{c}\right)^{-2}, & k>k_{c}\end{cases}
$$

where $k_{c}$ is a specified cut-off wavenumber. The filtered density distribution is then transferred back to physical space $\boldsymbol{x}$. The final result of the initial density distribution $\rho_{\sigma}(\boldsymbol{x}, 0)$ is a smoothed "double-delta" distribution with two peaks at $\rho_{\sigma}=\rho_{\sigma}^{\max }$ and $\rho_{\sigma}=\rho_{\sigma}^{\min }$.

(4) This initialization procedure is repeated for each species.

It should be noted that the smooth "double-delta" initial density fields generated by using the above initialization procedure are completely decoupled with the initial velocity fields $\boldsymbol{u}_{\sigma}(\boldsymbol{x}, t=0)$. Consequently the initial density fields so generated as passive scalars are not solutions of the Navier-Stokes equations.

\subsection{Decaying homogeneous isotropic turbulence}

Decaying homogeneous isotropic turbulence (DHIT) in a three-dimensional cube with periodic boundary conditions is a canonical test case to study turbulence with direct numerical simulation (DNS). A random and divergence free initial velocity field $\boldsymbol{u}$ is generated with a specified energy spectrum. Without external forcing, the flow decays to the quiescent state ultimately. The interested quantities in DHIT are moments of the energy spectrum $\tilde{E}(\boldsymbol{k}, t)$ with respect to the wave-vector $\boldsymbol{k}$, among which, the kinetic energy $K$ and the dissipation rate $\varepsilon$ are the lowest order moments of interest.

The energy spectrum $\tilde{E}(\boldsymbol{k}, t)$ in DHIT evolves as

$$
\partial_{t} \tilde{E}(\boldsymbol{k}, t)=-\tilde{T}(\boldsymbol{k}, t)-2 \nu k^{2} \tilde{E}(\boldsymbol{k}, t),
$$

where $\boldsymbol{k}$ is the wave vector $(k=|\boldsymbol{k}|$ is the wave number) and $\tilde{T}(\boldsymbol{k}, t)$ represents the nonlinear energy transfer between modes [6]. The kinetic energy $K$ and 
the dissipation rate $\varepsilon$ are given by

$$
K(t)=\int \tilde{E}(\boldsymbol{k}, t) d \boldsymbol{k}, \quad \varepsilon(t)=2 \nu \int k^{2} \tilde{E}(\boldsymbol{k}, t) d \boldsymbol{k} .
$$

It has been long observed that, after a short initial transient period of time, the kinetic energy and the dissipation rate exhibit a power-law decay:

$$
K(t) / K_{0} \sim\left(t / t_{0}\right)^{-n}, \quad \varepsilon(t) / \varepsilon_{0} \sim\left(t / t_{0}\right)^{-(n+1)},
$$

where $K_{0}$ and $\varepsilon_{0}$ are the values of $K(t)$ and $\varepsilon(t)$ at the reference time $t=$ $t_{0}=K_{0} / \varepsilon_{0}$, respectively. In turbulence, the relevant length scales are the Kolmogorov length scale

$$
\eta=\left(\frac{\nu^{3}}{\varepsilon}\right)^{1 / 4},
$$

which is the characteristic length of the smallest eddies, and the transverse Taylor microscale length

$$
\lambda=\sqrt{\frac{20 \nu K}{\varepsilon}},
$$

which defines the Taylor microscale Reynolds number:

$$
\operatorname{Re}_{\lambda}=\frac{u_{\mathrm{rms}} \lambda}{\nu}=K \sqrt{\frac{20}{3 \nu \varepsilon}},
$$

where $u_{\mathrm{rms}}=\sqrt{2 K / 3}$ is the root mean square (rms) of the velocity field $\boldsymbol{u}$.

In all of our simulations, we use the following initial energy spectrum for both species

$$
\tilde{E}_{0}(k)= \begin{cases}A k^{4} e^{-b k^{2}}, & k \in\left[k_{\min }, k_{\max }\right], \\ 0, & k \notin\left[k_{\min }, k_{\max }\right],\end{cases}
$$

where $A$ and $b$ are positive constants. The above initial energy spectrum is used to generate the random and divergence-free momentum fields for both species, which in turn are used to generate density and velocity fields for both species according to the procedure described in Sec. 4.1. The barycentric velocity $\boldsymbol{u}=\sum_{\sigma} x_{\sigma} \boldsymbol{u}_{\sigma}$ naturally satisfied the above energy spectrum initially.

\section{$5 \quad$ Results and Discussions}

We will report our numerical results for decaying homogeneous isotropic turbulence simulations using the LBE model for binary mixture. The simulations were conducted on a cube of system size $N^{3}$, with various values of $N$. There are many adjustable parameters pertinent to fluid properties in the

LBE model, as described in Sec. 3. We fix the following parameters in the 
simulations. First, the relaxation rates $\left\{s_{i}\right\}$ for both species $\mathrm{A}$ and $\mathrm{B}$, are the same. Specifically, $s_{\nu}=200 / 101$ so that the viscosity for both species, A and $\mathrm{B}$, is $\nu=1 / 600$ (in units of $\delta_{x}=\delta_{t}=1$ ), the relaxation rate for the bulk-viscosity $s_{\zeta}=6 / 5$, consequently $\zeta=5 / 27$, the diffusion relaxation rate $s_{D}=6 / 5$, and all other relaxation rates are set to 1 . In addition, the molecular weight $m_{\mathrm{A}}=1$ and the parameter $\varphi_{\mathrm{A}}=1$ for the species $\mathrm{A}$. We vary the B-species molecular weight $m_{\mathrm{B}}$ and the parameter $\varphi_{\mathrm{B}}$. Also the averaged particle number density ratio of the two species is fixed at 1, such that the averaged mass density ratio is equal to the ratio of the molecular weights, $m_{\mathrm{A}} / m_{\mathrm{B}}$. By varying $\varphi_{\mathrm{B}}=m_{\mathrm{A}} / m_{\mathrm{B}}$, we can adjust the speed of sound and the self diffusion coefficient $D_{\mathrm{B}}$ for species $\mathrm{B}$, and the mutual diffusion $D_{\mathrm{AB}}$.

\subsection{Scalar dynamics}

The first set of our simulations is to test the diffusion dynamics in the system. Because the lattice Boltzmann equation is restricted to the near incompressible flows with a practical Mach number limit of $\mathrm{Ma}<0.3$, we should expect that the magnitude of the velocity difference $\left\|\boldsymbol{u}_{\sigma}-\boldsymbol{u}_{\varsigma}\right\|$ is a quantity smaller than the species velocity magnitude $\left\|\boldsymbol{u}_{\sigma}\right\|$, consistent with the incompressibility. Therefore we expect our results to confirm the previous results obtained by using the incompressible Navier-Stokes equation and a passive scalar for diffusion, although our approach is an active scalar one. Because $\left\|\boldsymbol{u}_{\sigma}-\boldsymbol{u}_{\varsigma}\right\|$ is small, we therefore expect the dynamics of $\phi$ to be dominated by the diffusion except in the initial stage.

To measure the self-diffusion coefficients $D_{\sigma}$ in the LBE model, we first carried out a $1 \mathrm{D}$ simulation. The system size is $N_{x} \times N_{y} \times N_{z}=100 \times 10 \times 10$ and periodic boundary conditions are applied to all three directions. The initial density distribution is sinusoidal along the $x$ direction:

$$
\begin{aligned}
& \rho_{\mathrm{A}}(x, 0)=\bar{\rho}_{\mathrm{A}}\left[1+\delta_{\mathrm{A}} \sin \left(k_{x} x\right)\right], \\
& \rho_{\mathrm{B}}(x, 0)=\bar{\rho}_{\mathrm{B}}\left[1-\delta_{\mathrm{B}} \sin \left(k_{x} x\right)\right],
\end{aligned}
$$

where $\bar{\rho}_{\mathrm{A}}=0.64, \bar{\rho}_{\mathrm{B}}=1.15, \delta_{\mathrm{A}}=\delta_{\mathrm{B}}=0.001$, and $k=2 \pi / N_{x}$. With a constant $\boldsymbol{u}=\left(u_{x}, 0,0\right)$, the density $\rho_{\mathrm{A}}$ and $\rho_{\mathrm{B}}$ have the following solution:

$$
\begin{aligned}
& \rho_{\mathrm{A}}(x, t)=\bar{\rho}_{\mathrm{A}}\left[1+\delta_{\mathrm{A}} \sin \left(k_{x}\left(x-u_{x} t\right)\right)\right] e^{-D_{\mathrm{A}} k_{x}^{2} t}, \\
& \rho_{\mathrm{B}}(x, t)=\bar{\rho}_{\mathrm{B}}\left[1-\delta_{\mathrm{B}} \sin \left(k_{x}\left(x-u_{x} t\right)\right)\right] e^{-D_{\mathrm{B}} k_{x}^{2} t},
\end{aligned}
$$

Therefore, the evolution of the density variation can provide measurements of the self-diffusion coefficient $D_{\sigma}$ and the non-Galilean invariance effect. Figure 1 shows the measured self-diffusion coefficient $D_{\sigma}$ as a function of $s_{D}$ and $\varphi_{\sigma}=$ 
$m_{\mathrm{A}} / m_{\mathrm{B}}=1$ and $1 / 2$. The measured values of $D_{\sigma}$ agree with the analytic result of Eq. (26) at least three significant digits.

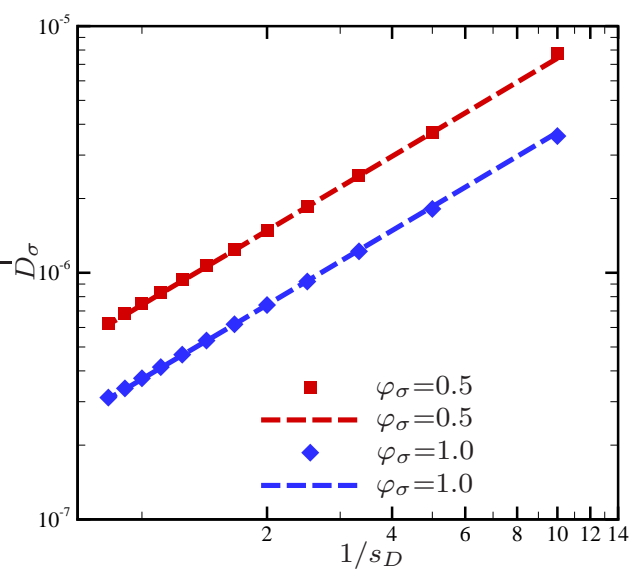

Fig. 1. Dependence of the self-diffusion coefficient $D_{\sigma}$ on the relaxation rate $s_{D}$ and $\varphi_{\sigma}$. The symbols are the values measured from the numerical simulations and the dashed lines are the results given by Eq. (26).

We next present the results of the scalar dynamics in the decaying homogeneous isotropic turbulence (DHIT) in 3D. The system size is $N^{3}=177^{3}$. We first use the passive scalar approach in order to reproduce the existing results [4]. We choose $m_{\mathrm{A}}=m_{\mathrm{B}}$ - the case closest to passive scalar approach. The initial divergence-free velocity fields for DHIT are generated with the energy spectrum of Eq. (46) and the following parameters: $u_{\mathrm{rms}}=0.0391$, $A=2.53 \cdot 10^{-5}, b=0.14,\left[k_{\min }, k_{\max }\right]=[1,14]$, and consequently $\operatorname{Re}_{\lambda}=12.4$. As for the density fields, we use the passive-scalar initialization procedure, described in Sec. 4.1, to initialize the density fields with the following parameters: $k_{s}=1, k_{0}=1$, and $k_{c}=2$; and $\rho_{\sigma}^{\min }=0.4$ and $\rho_{\sigma}^{\max }=0.6$. Figure 2 shows the result of the probability distribution function (PDF) of the concentration difference $\phi=\left(x_{\mathrm{A}}-x_{\mathrm{B}}\right)$. The PDF $P(\phi)$ begins with the initial distribution is close to a "double-delta" as shown in Fig. 2 and since there is not external forcing term (those previously discussed due to non-homogeneous local concentrations are actually driven by the definition of total mixture pressure and mixture viscosity), then evolves to a "single-delta" function, i.e., to the homogeneous equilibrium condition. This case is obviously different from that considered in other calculations [4], where the final steady-state distribution is sustained by (and highly depending on) the adopted forcing term.

We also simulate active scalar dynamics in DHIT. The initial momentum fields for DHIT are generated as the velocity fields in in the previous case of passive scalar dynamics. Then the density and velocity fields for each species are consistently generated with the active-scalar initialization procedure described in Sec. 4.1. The molecular mass ratios used in the simulations are $m_{\mathrm{B}} / m_{\mathrm{A}}=1$ and $m_{\mathrm{B}} / m_{\mathrm{A}}=8$. The results are shown in Fig. 3. In both cases of $m_{\mathrm{B}} / m_{\mathrm{A}}=1$ and 8 , the initial PDF $P(\phi, 0)$ is a Gaussian distribution centered at the 


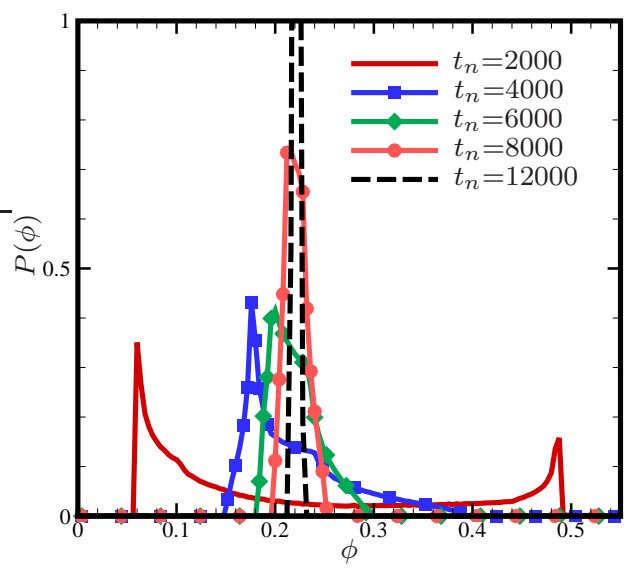

Fig. 2. The dynamics of the $\operatorname{PDF} P(\phi, t)$ with the passive scalar initialization for the density fields. $m_{\mathrm{A}}=m_{\mathrm{B}}=1$.

asymptotic value of $\phi, \phi_{\infty}=\left(m_{\mathrm{A}}-m_{\mathrm{B}}\right) /\left(m_{\mathrm{A}}+m_{\mathrm{B}}\right)$, with a very broad width, indicating large variance of $\phi$ initially. The width of $P(\phi, t)$ shrinks monotonically as it evolves in time. Our results show that, independent of initial state, the PDF $P(\phi, t)$ evolves to a "single-delta" function eventually, as expected.
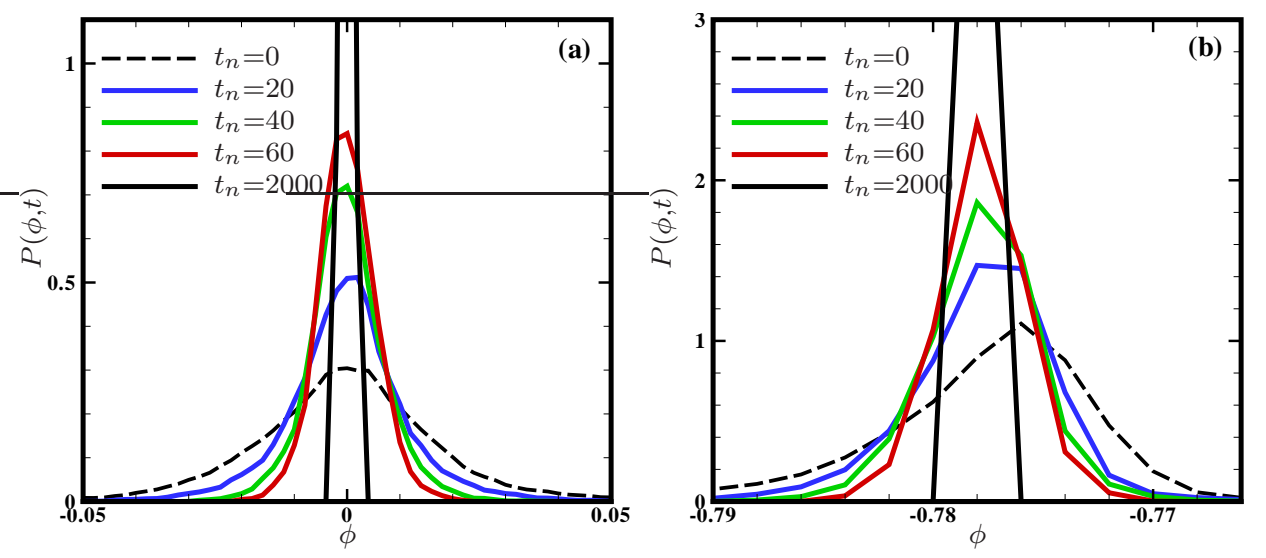

Fig. 3. PDF Dynamics of the mass concentration difference $\phi=\left(\rho_{\mathrm{A}}-\rho_{\mathrm{B}}\right) / \rho$. The PDFs have been normalized by a factor of $10^{6}$. (a) $m_{\mathrm{B}} / m_{\mathrm{A}}=1$ and (b) $m_{\mathrm{B}} / m_{\mathrm{A}}=8$.

\subsection{Decaying homogeneous isotropic turbulence}

Before we present the results for the decaying homogeneous isotropic turbulence, we must identify the appropriate energy for the system. For a binary mixture, there are two energies: the total kinetic energy $E$ based on the barycentric sum of the kinetic energies of each species and the kinetic energy of the barycentric velocity, and the former is not less than the latter in general:

$$
E=\frac{1}{2} \sum_{\sigma} x_{\sigma} u_{\sigma}^{2} \geq \frac{1}{2} u^{2}=K
$$


To identify the difference between $E$ and $K$, we perform a simulation with the system size of $N^{3}=45^{3}$, and the following parameters for the initial velocity fields: $u_{\mathrm{rms}}=0.0319, A=3.5926 \cdot 10^{-5}, b=0.14,\left[k_{\min }, k_{\max }\right]=$ $[2,4], \operatorname{Re}_{\lambda}=13.38$. In this case, the particle mass ratio is $m_{\mathrm{B}} / m_{\mathrm{A}}=9$. In Fig. 4 we show the total and the barycentric kinetic energies, normalized by the initial value $K_{0}$ of the barycentric kinetic energy, and the corresponding dissipation rates. Clearly, the mixture kinetic energy $E(t)$ quickly relaxes to the total kinetic energy $K(t)$, because the rotational energy, which accounts the difference between $E(t)$ and $K(t)$, dissipates quickly in the system. We also observed that the dissipation rate corresponding to $E(t)$ relaxes slower than $E(t)$ to the dissipation rate corresponding to $K(t)$, because the rotational effect has been magnified by $k^{2}$ for the dissipation rate. Nevertheless, this effect due to the rotational energy does not affect the decay exponent $n$ of the dissipation rate, as clearly shown in Fig. 4. Therefore, we will use the barycentric velocity $\boldsymbol{u}$ to compute kinetic energy in what follows.

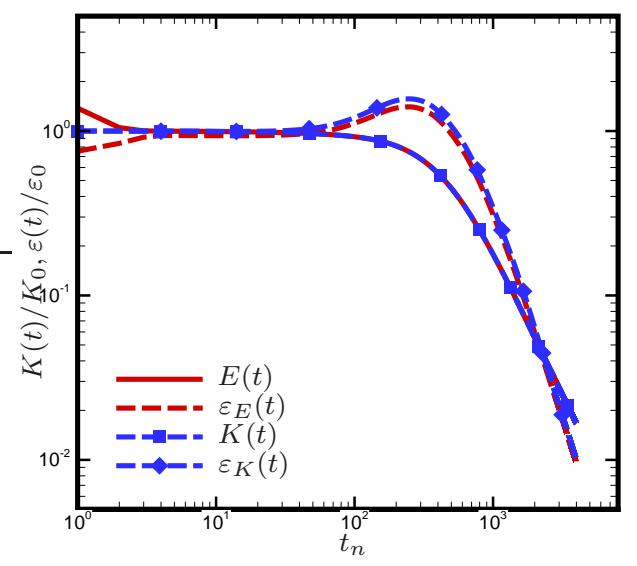

Fig. 4. Decay of the total kinetic energy $E(t)$ and the kinetic energy of the barycentric velocity $K(t)$, and the corresponding dissipation rates $\varepsilon_{E}(t)$ and $\varepsilon_{K}(t)$.

We performed a number of realizations for the DHIT simulations, compiled together in Table 1. In all the realizations, the molecular weight ratio is fixed at $m_{\mathrm{B}} / m_{\mathrm{A}}=9$, the mutual diffusion coefficient is $D_{\sigma \varsigma}=0.056$, and the viscosity is $\nu=1 / 600$. Each realization starts with different random initial conditions. The amplitude $A$, the width $b$ and the spectral range $\left[k_{\min }, k_{\min }\right]$ of the initial energy spectrum, the rms value of the initial velocity field $u_{\mathrm{rms}}$, and the initial Taylor-microscale Reynolds number $\mathrm{Re}_{\lambda}$ for each realization are also given in Table 1. The realizations marked with "*" are performed with the passive scalar approach by assuming $\phi$ to its constant averaged value in the momentum equation, as discussed in Sec. 3. For each realization, we measure the exponent $n$ from both the kinetic energy $K(t)$ and the dissipation rate $\varepsilon(t)$, and compute the averaged value of these two measurements for $n$. The values of $n$ are measured when both $K(t)$ and $\varepsilon(t)$ reach the stage of (nearly) power-law decay. The averaged values of $n$ are given in Table 1. Clearly, the values $n$ are larger for smaller values of $\mathrm{Re}_{\lambda}$. This observation is consistent 
with existing experimental and numerical results. We compile in Table 2 the exponent $n$ in comparison with existing data.

Table 1

The decay exponent in DHIT obtained in a number of realizations. The exponent $n$ is the mean value between those measured from $K$ and $\varepsilon$, namely $n_{K}$ and $n_{\varepsilon}$ respectively. The runs marked with $*$ are initialized with passive-scalar initial conditions.

\begin{tabular}{l|l|c|c|c}
\hline \hline No. & \multicolumn{1}{|c|}{ Parameters } & $n_{K}$ & $n_{\varepsilon}$ & $n$ \\
\hline 1 & $N^{3}=63^{3},\left[k_{\min }, k_{\max }\right]=[1,4]$ & 1.818 & 1.725 & 1.772 \\
$2^{*}$ & $A=3.4293 \cdot 10^{-5}, b=0.14$ & 1.787 & 1.874 & 1.831 \\
3 & $u_{\mathrm{rms}}=0.0327, \mathrm{Re}_{\lambda}=14.16$ & 1.877 & 1.794 & 1.836 \\
$4^{*}$ & $K_{0}=1.6020 \cdot 10^{-3}, \varepsilon_{0}=5.1209 \cdot 10^{-5}$ & 1.909 & 1.910 & 1.910 \\
5 & & 1.906 & 1.763 & 1.835 \\
$6^{*}$ & & 1.576 & 1.595 & 1.585 \\
\hline 7 & $N^{3}=63^{3},\left[k_{\min }, k_{\max }\right]=[2,4]$ & 1.581 & 1.792 & 1.686 \\
$8^{*}$ & $A=3.4407 \cdot 10^{-3}, b=0.56$ & 1.793 & 1.935 & 1.864 \\
9 & $u_{\mathrm{rms}}=0.0558, \mathrm{Re}_{\lambda}=29.11$ & 1.738 & 1.635 & 1.686 \\
$10^{*}$ & $K_{0}=4.6729 \cdot 10^{-3}, \varepsilon_{0}=1.0308 \cdot 10^{-4}$ & 1.780 & 1.849 & 1.815 \\
\hline 11 & $N^{3}=123^{3},\left[k_{\min }, k_{\max }\right]=[1,8]$ & 1.566 & 1.428 & 1.497 \\
$12^{*}$ & $A=1.1727 \cdot 10^{-4}, b=0.14$ & 1.625 & 1.572 & 1.599 \\
13 & $u_{\mathrm{rms}}=0.0840, \mathrm{Re}_{\lambda}=26.76$ & 1.291 & 1.369 & 1.330 \\
$14^{*}$ & $K_{0}=1.0575 \cdot 10^{-2}, \varepsilon_{0}=6.2480 \cdot 10^{-4}$ & 1.485 & 1.391 & 1.438 \\
15 & & 1.389 & 1.441 & 1.415 \\
$16^{*}$ & & 1.548 & 1.372 & 1.460 \\
\hline \hline 2
\end{tabular}

Table 2

Existing data for the exponent $n$. The present results in Table 1 are given as LBEDNS.

\begin{tabular}{c|c|r}
\hline \hline $\operatorname{Re}_{\lambda}$ & exponent $n$ & \multicolumn{1}{|c}{ Refs. } \\
\hline $0-30$ & $1.1-1.52$ & NS-DNS [57] \\
$10-50$ & $1.25-1.51$ & NS-DNS [58] \\
$4.4-5.4$ & $1.3-1.8$ & Exp. [59] \\
$28.37-43.85$ & $1.285-1.309$ & Exp. [60] \\
$2.3-22.5$ & $1.38-1.85$ & LBGK-DNS [30] \\
$14.16-29.11$ & $1.330-1.910$ & LBE-DNS \\
\hline \hline
\end{tabular}

Figure 5(a) shows a typical dynamics of the kinetic energy $K(t)$ and the 
dissipation rate $\varepsilon(t)$. The realization was carried out with a system size of $N^{3}=$ $135^{3}$ and 32,700 time steps. The parameters for the initial energy spectrum are $A=2.7985 \cdot 10^{-6}, b=0.14,\left[k_{\min }, k_{\max }\right]=[4,8]$, and $u_{\mathrm{rms}}=0.0090$, resulting in $K_{0}=1.2162 \cdot 10^{-4}$ and $\varepsilon_{0}=1.0731 \cdot 10^{-5}, \operatorname{Re}_{\lambda}=2.35$. The time $t$ is normalized by $t_{0}=n K_{0} / \varepsilon_{0} \approx 18.89$, assuming $n=5 / 3$. Figure 5 (a) clearly shows that both $K(t)$ and $\varepsilon(t)$ decay according to power-laws with the decaying exponents 1.60 and 2.66, respectively, in the late dynamics, i.e., after $t / t_{0}>100$.
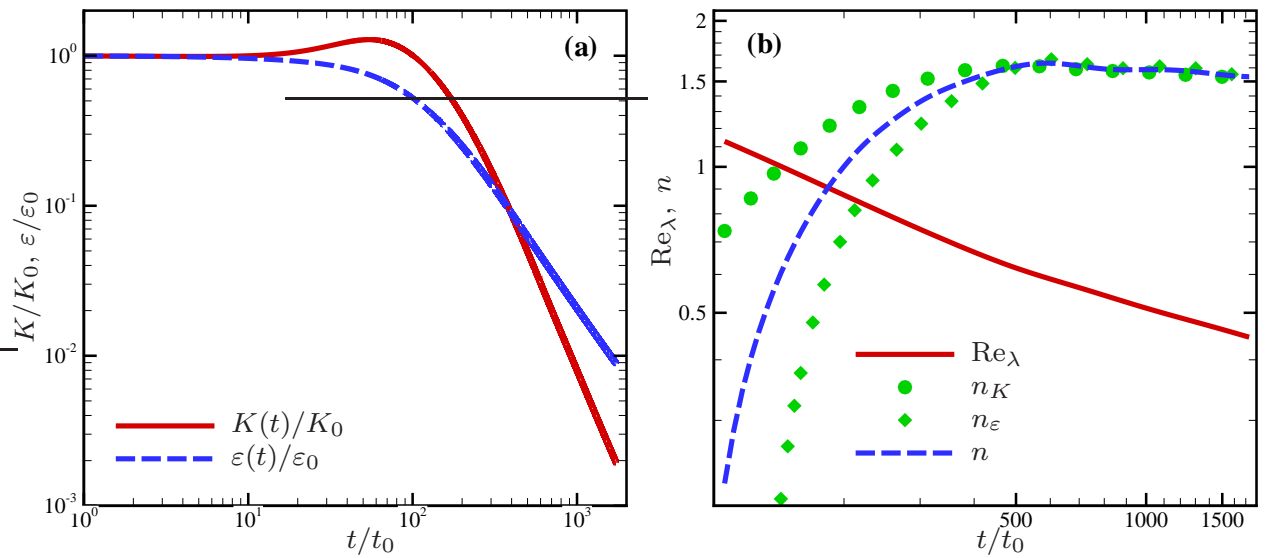

Fig. 5. The dynamics of (a) the kinetic energy $K(t)$ and the dissipation rate $\varepsilon(t)$ and (b) the Taylor micro-scale Reynolds number $\operatorname{Re}_{\lambda}(t)$ and the decay exponent $n(t) . N^{3}=135^{3}, m_{\mathrm{B}} / m_{\mathrm{A}}=9, K_{0}=1.2162 \cdot 10^{-4}, \varepsilon_{0}=1.0731 \cdot 10^{-5}, t_{0} \approx 18.89$ and $\operatorname{Re}_{\lambda}(0)=2.35$.

To demonstrate the dynamics of the decaying exponent $n$, in Fig. $5(\mathrm{~b})$ we show the dynamics of $n$ computed from both $K(t)$ and $\varepsilon(t)$, their average, and corresponding $\operatorname{Re}_{\lambda}(t)$ measured from $K(t)$ and $\varepsilon(t)$. Clearly, the values of $n$ measured from $K(t)$ and $\varepsilon(t)$ converge to their averaged value as $\operatorname{Re}_{\lambda}(t)$ decreases. When $n_{K}$ and $n_{\varepsilon}$ converge to their average value $n$ after $t / t_{0} \approx$ $500, \operatorname{Re}_{\lambda}(t)$ also shows power-law decay, which should be $\left(t / t_{0}\right)^{(1-n) / 2}$. The dimensionless Kolmogorov scale $\eta$ for this particular realization is always larger than $0.5 \delta_{x}$ at the time when the exponent $n$ is measured, ensuring that the flow is well resolved [6].

\section{Conclusions}

In this paper we have proposed a consistent lattice Boltzmann equation with baroclinic coupling between species dynamics and mixture dynamics in multispecies mixtures. The proposed lattice Boltzmann equation models the active scalar dynamics in mixtures and has the following distinctive features. First, it is directly derived from the linearized Boltzmann equations for mixtures. Second, it uses the multiple-relaxation-time collision model so that it has the 
flexibility of independent Reynolds and Schmidt numbers. In addition, it has better numerical stability. Thirdly, it satisfies the indifferentiability principle and therefore leads to consistent hydrodynamic equations for the mixture barycentric velocity.

The proposed LBE model is validated through the simulations of decaying homogeneous isotropic turbulence in three dimensions. We first simulate both active and passive scalar dynamics in decaying turbulence for mixtures. We also compute various statistical quantities and their decay exponents in decay turbulence. Our results agree well existing results for both scalar dynamics and decaying turbulence. Our results indicate that, in low Mach number flows, the diffusion velocity is rather small and does not have any substantial effects. This is consistent with physics for equilibrium flows at small Mach number and nearly zero Knudsen number. This situation will change when the Mach and Knudsen numbers increase and this will be the subject of our future investigation. Our future investigation will include forced turbulence and compressible turbulence modeled by kinetic schemes for mixture dynamics.

\section{Acknowledgments}

PA would like to thank Profs. Michele Calì and Romano Borchiellini of Politecnico di Torino for their support and encouragement. This work is supported by DOD under AFOSR-MURI Project "Hypersonic transition and turbulence with non-equilibrium thermochemistry" (Dr. J. Schmisseur, Program Manager), by NSF through Grant CBET-0500213, and by NASA LaRC through a C\&I grant via an NIA cooperation agreement grant NCC-1-02043. The computing resources available to the authors at Politecnico di Torino and Department of Computer Science of Old Dominion University are gratefully acknowledged.

\section{References}

[1] P. E. Dimotakis. Turbulent mixing. Annu. Rev. Fluid Mech., 37:329-356, 2005.

[2] C. Eckart. An analysis of the stirring and mixing processes in incompressible fluids. J. Mar. Res., 7:265-275, 1948.

[3] A. Kronenburg and R. W. Bilger. Modelling of differential diffusion effects in nonpremixed nonreacting turbulent flow. Phys. Fluids, 9(5):1435-1447, 1997.

[4] V. Eswaran and S. B. Pope. Direct numerical simulations of the turbulent mixing of a passive scalar. Phys. Fluids, 31(3):506-520, 1988. 
[5] G. Brethouwer, B.J. Boersma, M. B. J. M. Pourquie, and F. T. M. Nieuwstadt. Direct numerical simulation of turbulent mixing of a passive scalar in pipe flow. Euro. J. Mech. B/Fluids, 18(4):739-756, 1999.

[6] S. B. Pope. Turbulent Flows. Cambridge University Press, Cambridge, UK, 2nd edition, 2000.

[7] S. M. de Bruyn Kops, J. J. Riley, and G. Kosaly. Direct numerical simulation of reacting scalar mixing layers. Phys. Fluids, 13(5):1450-1465, 2001.

[8] C. Pantano, S. Sarkar, and F.A. Williams. Mixing of a conserved scalar in a turbulent reacting shear layer. J. Fluid Mech., 481:291-328, 2003.

[9] H. Kolera-Gokula and T. Echekki. Direct numerical simulation of premixed flame kernel-vortex interactions in hydrogen-air mixtures. Combust. Flame, 146(1/2):155-167, 2006.

[10] C. Pantano. Direct simulation of nonpremixed flame extinction in a methane-air jet with reduced chemistry. J. Fluid Mech., 514:231-270, 2004.

[11] M. V. Papalexandris. Numerical simulation of detonations in mixtures of gases and solid particles. J. Fluid Mech., 507(25):95-142, 2004.

[12] R. B. Bird, W. E. Stewart, and E. N. Lightfoot. Transport Phenomena. John Wiley \& Sons, New York, 1960.

[13] F. A. Williams. Combustion Theory. Benjamin/Cummings, Menlo Park, CA, 2nd edition, 1985.

[14] J. H. Irving and J. G. Kirkwood. The statistical mechanical theory of transport process. IV. The equations of hydrodynamics. J. Chem. Phys., 18(6):817-829, 1950.

[15] D. Z. Zhang, X. Ma, and R. M. Rauenzahn. Interspecies in momentum equations for dense binary particulate systems. Phys. Rev. Lett., 97:048301, 2006.

[16] X. Shan and H. Chen. Lattice Boltzmann model for simulating flows with multiple phases and components. Phys. Rev. E, 47:1815-1819, 1993.

[17] M. R. Swift, E. Orlandini, W. R. Osborn, and J. M. Yeomans. Lattice Boltzmann simulation of liquid-gas and binary fluid systems. Phys. Rev. E, 54:5041-5052, 1996.

[18] L.-S. Luo. Unified theory of the lattice Boltzmann models for nonideal gases. Phys. Rev. Lett., 81:1618-1621, 1998.

[19] L.-S. Luo. Theory of lattice Boltzmann method: Lattice Boltzmann models for nonideal gases. Phys. Rev. E, 62:4982-4996, 2000.

[20] L.-S. Luo and S. S. Girimaji. Lattice Boltzmann model for binary mixtures. Phys. Rev. E, 66:035301(R), 2002.

[21] L.-S. Luo and S. S. Girimaji. Theory of the lattice Boltzmann method: Twofluid model for binary mixtures. Phys. Rev. E, 67:036302, 2003. 
[22] P. Asinari. Viscous coupling based lattice Boltzmann model for binary mixtures. Phys. Fluids, 17(6):067102, 2005.

[23] P. Asinari. Semi-implicit-linearized multiple-relaxation-time formulation of lattice Boltzmann schemes for mixture modeling. Phys. Rev. E, 73(5):056705, 2006.

[24] D. d'Humières. Generalized lattice-Boltzmann equations. In B. D. Shizgal and D. P. Weave, editors, Rarefied Gas Dynamics: Theory and Simulations, volume 159 of Prog. Astronaut. Aeronaut., pages 450-458, Washington, D.C., 1992. AIAA.

[25] P. Lallemand and L.-S. Luo. Theory of the lattice Boltzmann method: Dispersion, dissipation, isotropy, Galilean invariance, and stability. Phys. Rev. E, 61:6546-6562, 2000.

[26] D. d'Humières, M. Bouzidi, and P. Lallemand. Thirteen-velocity threedimensional lattice Boltzmann model. Phys. Rev. E, 63:066702, 2001.

[27] D. d'Humières, I. Ginzburg, M. Krafczyk, P. Lallemand, and L.-S. Luo. Multiple-relaxation-time lattice Boltzmann models in three-dimensions. Philos. Trans. R. Soc. Lond. A, 360:437-451, 2002.

[28] P. Lallemand and L.-S. Luo. Theory of the lattice Boltzmann method: Acoustic and thermal properties in two and three dimensions. Phys. Rev. E, 68:036706, 2003.

[29] P. L. Bhatnagar, E. P. Gross, and M. Krook. A model for collision processes in gases. I. Small amplitude processes in charged and neutral one-component systems. Phys. Rev., 94:511-525, 1954.

[30] H. Yu, S. S. Girimaji, and L.-S. Luo. Lattice Boltzmann simulations of decaying homogeneous isotropic turbulence. Phys. Rev. E, 71:016708, 2005.

[31] H. Yu, S. S. Girimaji, and L.-S. Luo. DNS and LES of decaying isotropic turbulence with and without frame rotation using lattice Boltzmann method. J. Comput. Phys., 209(2):599-616, 2005.

[32] G. Erlebacher, M. Y. Hussaini, H. O. Kreiss, and S. Sarkar. The analysis and simulation of compressible turbulence. Theor. Comput. Fluid Dyn., 2(2):73-95, 1990.

[33] S. Lee, S. K. Lele, and P. Moin. Eddy shocklets in decaying compressible turbulence. Phys. Fluids A, 3(4):657-664, 1991.

[34] R. Samtaney, D. I. Pullin, and B. Kosović. Direct numerical simulation of decaying compressible turbulence and shocklet statistics. Phys. Fluids, 13(5):1415-1430, 2001.

[35] S. Pirozzoli and F. Grasso. Direct numerical simulations of isotropic compressible turbulence: Influence of compressibility on dynamics and structures. Phys. Fluids, 16(12):4386-4407, 2004. 
[36] J. O. Hirschfelder, C. F. Curtiss, and R. B. Bird. Molecular Theory of Gases and Liquids. Wiley, New York, 1954.

[37] S. Chapman and T. G. Cowling. The Mathematical Theory of Non-Uniform Gases. Cambridge University Press, Cambridge, UK, 3rd edition, 1970.

[38] J. H. Ferziger and H. G. Kaper. Mathematical Theory of Transport in Gases. North Holland, Amsterdam, 1972.

[39] L. C. Woods. An Introduction to the Kinetic Theory of Gases and Magnetoplasmas. Oxford University Press, Oxford, UK, 3 edition, 1993.

[40] S. Harris. An Introduction to the Theory of the Boltzmann Equation. Dover, Mineola, NY, 2004.

[41] I. Kolodner. On the application of the Boltzmann equations to the theory of gas mixtures. Phd thesis, New York University, New York, 1950.

[42] C. F. Curtiss and J. O. Hirschfelder. Transport properties of multicomponent gas mixtures. J. Chem. Phys., 17:550-555, 1949.

[43] E. P. Gross and M. Krook. Models for collision processes in gases: Smallamplitude oscillations of charged two-component systems. Phys. Rev., 102:593$604,1956$.

[44] E. P. Gross and E. A. Jackson. Kinetic models and the linearized Boltzmann equation. Phys. Fluids, 2(4):432-441, 1959.

[45] L. Sirovich. Kinetic modelling of gas mixtures. Phys. Fluids, 5:908-918, 1962.

[46] T. F. Morse. Kinetic model equations for a gas mixture. Phys. Fluids, 7:20122013, 1964.

[47] B. B. Hamel. Kinetic models for binary mixtures. Phys. Fluids, 8:418-425, 1965.

[48] L. Sirovich. Mixtures of Maxwell molecules. Phys. Fluids, 9:2323-2326, 1966.

[49] B. B. Hamel. Two-fluid hydrodynamic equations for a neutral, disparate-mass, binary mixtures. Phys. Fluids, 9:12-22, 1966.

[50] S. Ziering and M. Sheinblatt. Kinetic theory of diffusion in rarefied gases. Phys. Fluids, 9:1674-1685, 1966.

[51] E. Goldman and L. Sirovich. Equations for gas mixtures. Phys. Fluids, 10:19281940, 1967.

[52] J. M. Greene. Improved Bhatnagar-Gross-Krook model of electron-ion collisions. Phys. Fluids, 16:2022-2023, 1973.

[53] H. Grad. Principles of the kinetic theory of gases. In S. Flügge, editor, Handbuch der Physik, pages 204-294. Springer, Berlin, 1958.

[54] J. C. Maxwell. On the dynamical theory of gases. Philos. Trans. R. Soc., 157:26-78, 1866. 
[55] H. Grad. Theory of rarefied gases. In F. Devienne, editor, Rarefied Gas Dynamics, pages 100-138. Pergamon, London, 1960.

[56] P. Andries, K. Aoki, and B. Perthame. A consistent BGK-type model for gas mixtures. J. Stat. Phys., 106(5/6):993-1018, 2002.

[57] N. N. Mansour and A. A. Wray. Decay of isotropic turbulence at low Reynolds number. Phys. Fluids, 6(2):808, 1994.

[58] M.-J. Huang and A. Leonard. Power-law decay of homogeneous turbulence at low Reynolds numbers. Phys. Fluids, 6(11):3765-3775, 1994.

[59] M. R. Smith, R. J. Donnelly, N. Goldenfeld, and W. F. Vinen. Decay of vorticity in homogeneous turbulence. Phys. Rev. Lett., 71:2583-2586, 1993.

[60] M. S. Mohamed and J. C. LaRue. The decay power law in grid-generated turbulence. J. Fluid Mech., 219:195-214, 1990. 